\title{
Interactive Entertainment, Virtual Motion Training and Brain Ergonomy
}

\author{
Sara Invitto, Chiara Faggiano, Silvia Sammarco \\ Human Anatomy and Neuroscience Lab \\ Department of Biological and \\ Environmental Science and Technology \\ University of Salento \\ Lecce, Italy \\ sara.invitto@unisalento.it \\ chiarafaggiano0@gmail.com \\ silvia.sammarco@yahoo.it
}

\author{
Valerio De Luca, Lucio T. De Paolis \\ Augmented and Virtual Reality Lab \\ Department of Engineering for Innovation \\ University of Salento \\ Lecce, Italy \\ \{valerio.deluca, lucio.depaolis\}@unisalento.it
}

\begin{abstract}
Aim of this work was to study the perception of affordances in VR according to a cognitive neuroscience. In order to investigate this topic we focused our analysis on the perception of affordances during a game, the Leap Motion Controller (LM). The sample was composed of 10 university students matched by age and sex. The subjects performed in Thinking training and in Immersive Affordance Condition (a virtual training with LM and a Real training). After each training the subject had to perform a recognition task. During the task ERP components were measured through EEG. The results highlighted that, during the LM training, attentional components changed. In the occipital lobes, which is entrusted to visual sensory, we got increased latencies; on the contrary, in frontal lobe, where the brain mainly activates for attention and action planning, we got decreased latencies.

Keywords - Interactive Game, Virtual Training, Event Related Potentials, Cognitive Neuroscience
\end{abstract}

\section{INTRODUCTION}

The concept of affordance was first introduced by Gibson [1], who observed that the dynamical pattern of the optic flow can be used to guide navigation reactively through the environment. He used the term affordance to refer to the fact that visual perception of the environment is not just passive perception of objects as such, but direct perception of the potential actions that the perceiver can carry out with them without the need for high-level processes such as reasoning about object properties [2]. In the realm of manipulation, for example, a person seeing an object would not necessarily only perceive colours, shapes and so on, but firstly and foremost also directly perceive the object's "graspability", "liftability" and so on [3]. The affordances of any given object depend not only on the object, but also on the embodiment (in particular the actuators) of the perceiving agent. A bottle, for example, affords grasping for humans but not for dogs (for which it might afford a biting action) or ants. A key aspect of the concept of affordance is the reactive nature of the resulting sensorimotor processing that tends to trigger or prime action in an automatic fashion (although we will later see that this tendency can be strongly modulated by the context and goals of the agent).

In some of the recent literature, the concept of affordance has been extended beyond the Gibsonian interpretation to consider the brain representations of affordances (the possible sensorimotor interactions offered by objects, see for instance) [4]. These representations encode both the features of the objects needed to act on them (e.g., the size and location of the object) and the relation between the objects and the agent's body (e.g., that an object is within reach or in contact with a hand). Several researchers consider that the Gibson's ecological framework is a promising functional approach for defining the reality of experience in relation to the problem of designing virtual environments [5]. For example, the perception of affordances could be a potential tool for sensorimotor assessment of physical presence, that is the feeling of being physically located in a virtual place [6]. Therefore, Bringoux investigated the walk through a virtual aperture of variable widths. In the case of presence, the subject's body orientation, while walking, was hypothesized to be adapted to the aperture width: it indicated that the locomotor postural patterns of subjects having to walk through a virtual aperture strongly resemble those of subjects who have to walk through a real aperture [7]. For most subjects, a behavioral transition from frontal walking to body rotation was observed as the width of the virtual aperture decreased. Finally, researchers designed a conceptual model in order to evoke affordances in Virtual Environments (VE) via sensory-stimuli substitution. Such a model can potentially guide VE designers in generating more ecologically valid designs [5]. Currently, the use of Virtual Reality (VR) technology in the study of the cognitive process in the human brain has been reported in some Event Related Potentials (ERP) literature. An event-related potential is the measured brain response that is the direct result of a specific sensory, cognitive, or motor event [8]. Therefore, the mechanism of cognitive processing in the human brain could be better studied by using virtual reality technology to construct realistic experimental scenarios. In a recent ex- 
periment [9], through the presentation of traffic signs with correct or incorrect background colors in a virtual reality traffic environment, the cognitive processing in the human brain was studied with the event-related potential (ERP) method. The results showed that simpler contents and larger contrast between the background colors and foreground colors of traffic signs would make the human brain response faster. According to this approach, some studies believes that AR could be a candidate to produce better adherence to correct procedures by virtue of increasing motivation [10]. Therefore, the purpose of this article was to study the perception of affordances in VR through electrophysiological variables (ERP), according to a neuroscientific aspect of cognition. In order to investigate this topic empirically, we chose to focus our analysis on the perception of affordances during an interactive experience with the Leap Motion Controller [11]. In accordance to our model, the use of new Information and Communication Technologies within Science Education is linked to date a series of interactive software that can play at a very high level of technology, making them extremely compelling in their interaction. These software applications are accessible by the user to play and to learn some objects and have expanded the possibilities for experimentation of categories in which people can experience situations in virtual way.

\section{Method}

\section{A. Participants}

Our Sample was composed by 10 university students matched by age and sex ( 5 men and 5 women). The sample of recruited volunteers had normal hearing, normal or corrected to normal vision and a right manual dominance. Subjects recruited had not previous experience of EEG cognitive task. The subjects performed in Baseline Condition Study (Thinking training) and in Immersive Affordance Condition (a virtual training with Leap Motion and a Real training). None of them had previously taken part in experiments like these. All participants gave written informed consent according to Helsinki declaration.

\section{B. Measurements and Stimuli}

Data sheet of the subject (age, sex), Object with grasping's Affordance (cup, glasses, scissors, pot handle, mouse, fork, pen); Behavioral Task on Image Recognition (recorded through E-Prime 2.0 Presentation), EEG Recording, Leap Motion Training.

1) EEG: During the images presentation task we recorded an EEG 16 Channels of Brain AMP - Brain Vision Recorder. We Considered in EEG tracks, the Event Related Potentials (ERP) for averaged waves for Grasping Object and No Grasping Object. During the computer supported attention task, EEG was recorded by 16 surface recording electrodes, belonging to the Brain Vision Recorder apparatus (Brain Vision, EMS Medical- Italy). A further electrode was positioned above the right eyebrow for electro-oculogram (EOG) recording. The ERP's analysis was obtained through the Brain Vision
Analyzer. Time off-line analysis was from $100 \mathrm{~ms}$ prestimulus to $500 \mathrm{~ms}$ poststimulus with $100-0$ baseline-correction. Thereafter, trials with blinks and eye movements were rejected on the basis of horizontal electro-oculogram with a ICA component analysis. An artifact rejection criterion of $60 \mathrm{~V}$ was used at all other scalp sites to reject trials with excessive EMG or other noise transients. Sampling rate was $256 \mathrm{~Hz}$. After a transformation and a re-segmentation of data with Brain Vision Analyzer, the artifact-free EEG tracks correspondent to the affordance object, marked by the motor response, were averaged in each case to extract the main waveforms, the N1 in the $120-175 \mathrm{msec}$ time range, the $\mathrm{P} 1$ in the $175-250 \mathrm{msec}$ time range and the P3 component in the $260-400 \mathrm{msec}$ time interval, according to literature. We performed a semiautomatic Peak Detection with the mean of the maximum area for the different components of ERP's waves.

2) Leap Motion: The Leap Motion Controller [11] is a small, easy to use and low-cost device designed to capture the movements of human hands and fingers. It can be plugged to a USB port of a Windows, Linux or Mac computer (Figure $1)$.

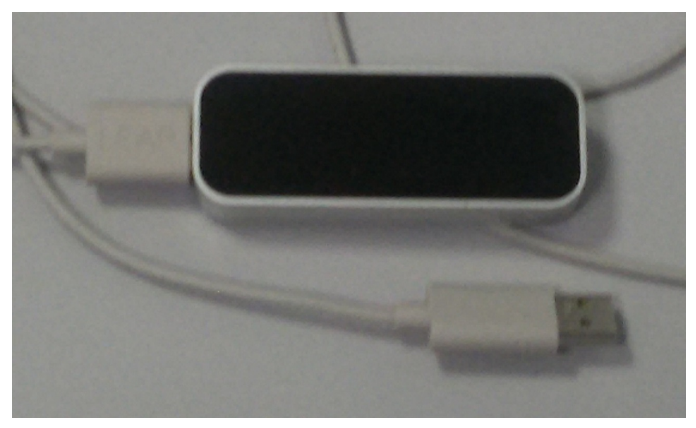

Figure 1. The Leap Motion Controller

It has a maximum field of view of $150^{\circ}$, which forms an inverted pyramid with the vertex placed in the middle of the device: the effective detection range roughly extends from 0.25 to $60 \mathrm{~cm}$ above the device. As declared by the manufacturer, the controller has a maximum frame rate of $300 \mathrm{fps}$ and an accuracy of $0.01 \mathrm{~mm}$ in the fingertip position detection. However, some experimental tests [12], conducted on the Leap Motion preliminary version, proved the theoretical accuracy of $0.01 \mathrm{~mm}$ cannot be actually achieved, even though the measured average accuracy of $0.7 \mathrm{~mm}$ is still quite good among gesture-based user interfaces, especially if compared to other low cost devices such as Microsoft Kinect. While the Leap Motion controller focuses only on hand recognition, the Microsoft Kinect sensor provides a full-body gesture recognition but at a lower resolution. Some experimental tests [13] showed Kinect is not able to detect precise hand and finger gestures: based on the sensor distance, the error in depth measurement increases from a few millimeters to roughly 4 $\mathrm{cm}$; the depth accuracy has a standard deviation of about 1.5 $\mathrm{cm}$.

Motion information is discretized into a sequence of states, which the API represents as frames. Each frame contains 
specific information characterizing hands and fingers in a particular state. Furthermore, the API recognizes some particular movement combinations, represented as some entities called Gestures.

Version 2.0 of the API solves also occlusion problems introducing the so-called Skeletal Tracking Model [14], based on an internal model of the human hand. This expedient improves the persistence in the detection of hands and fingers and consequently also the usability of most Leap Motion-based applications.

The Leap Motion app store [15] collects several apps developed with the Leap Motion SDK. In our study we considered the Playground app [16], which consists in three training scenarios providing a quick introduction to the device tracking capabilities. The first scene invites the user to put his/her hands over the controller and shows on the screen a ghostly and sparkling representation which reproduces hand's movements.

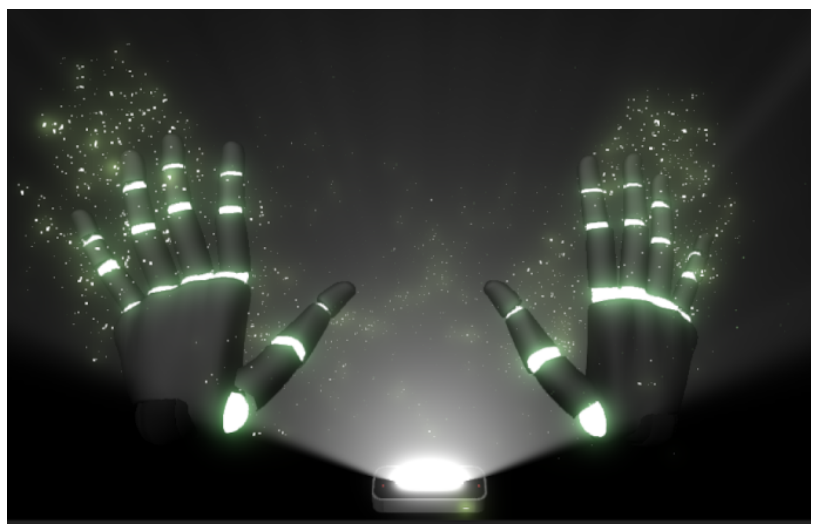

Figure 2. The Leap Motion Playground app

The second and the third scenes help the user in getting familiar with the interaction with virtual objects in a 3D space. The aim of these scenes is to reduce the cognitive friction by making the user experience progressively more intuitive.

For our experiments we chose the first Playground scene, where only the user hands are reproduced on the screen: in this way, the user can concentrate on using real objects belonging to the affordance set we will present in section II-C.

\section{Procedure and Task}

Our study/experiment consisted in an analysis of affordances perception in VR through electrophysiological variable (ERP).The task in Baseline condition shows, in a pseudo random way, images (pictures in $2 \mathrm{D}$ presentation, with the software e-Prime 2.0) like Colored Frames, Objects, Grasping Object (with affordance of motion i.e., glasses, cup, scissors, mouse, pen, fork) (Figure 3).

Subjects were seated in a comfortable chair $100 \mathrm{~cm}$ from the display monitor; their midsagittal plane was aligned with the center of the screen and with the center of Leap Motion. Subjects performed 3 different tasks during the experiment (Figure 4): Training Task A in which they were asked to

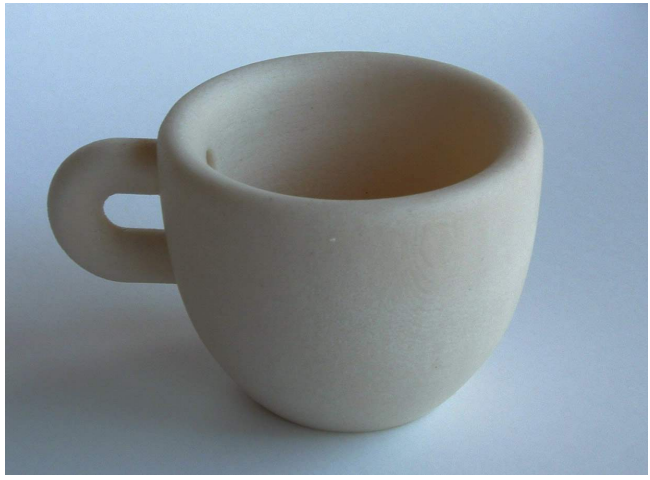

Figure 3. Example of an object with Grasping Affordance

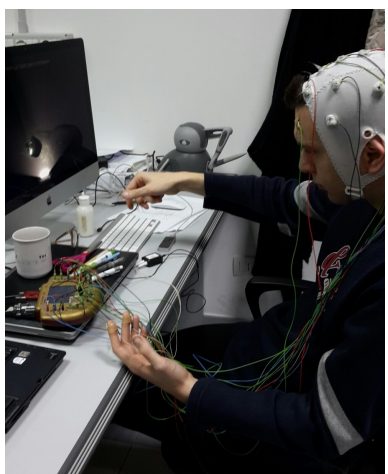

Figure 4. Subject during a Virtual Training with Leap Motion

think using the objects with affordance of grasping (the objects where positioned in front of the subject, on the table); Training Task B in which they used the objects while interacting with the Leap Motion Playground app (they had a visual feedback on the screen); and Task Training $\mathrm{C}$ in which they really used the objects. Each Training Task Condition had a duration of 2 minutes.

Conditions in each Training Task were pseudorandom. After each Training the subject had to perform an E-prime experiment in which he had to recognise, between various objects and colored frames, the objects he had previous seen during the training. The triggers of Affordance Images were used for ERP Segmentation Anaysis.

The images of Recognition Task were selected through a repertoire of neutral images (colored squares on a light background), non target images (animals, everyday objects) and target images (the same Grasping Objects used in the previous Training). All stimuli had a dimension of $240 \mathrm{x}$ 210 pixels, and have been displayed centrally on a light gray background and to the same level of brightness on the computer monitor. The Task was administered via the E-prime software 2.0, an application of software tools Psychology, Inc. The task paradigm was a Go-NoGo presentation (Figure 5). Each trial, consisting in a single type of target alternated randomly background-color, has lasted 600 seconds, with a stimulus duration of $2000 \mathrm{~ms}$ and $1000 \mathrm{~ms}$ interstimulus duration. The participants were instructed to stand upright with 


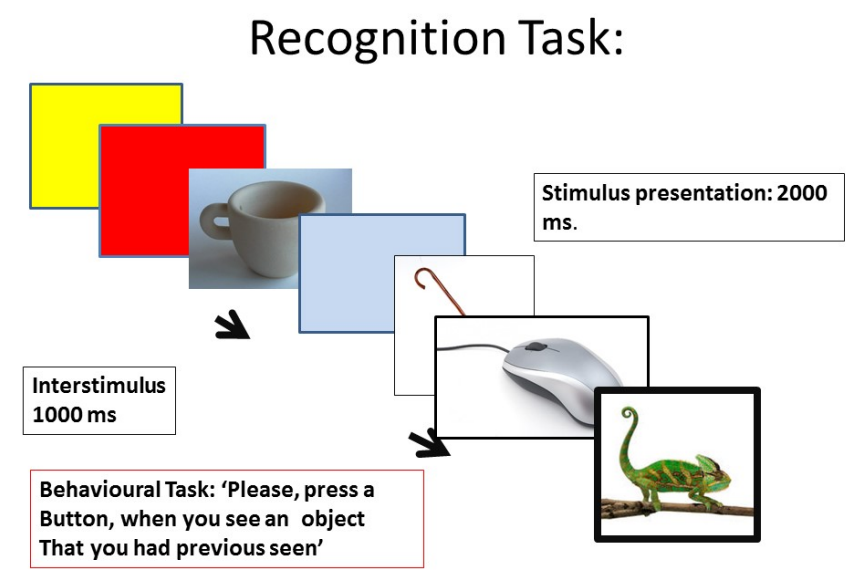

Figure 5. Recognition Task: E-Prime presentation with a Go-NoGo Task

ca $75 \mathrm{~cm}$ between the front edge of the chair and the floor of the computer screen. The following instruction message was showed to each user: "Please click a button when you see an element which has been previously imagined or manipulated".

\section{Statistical Analysis and Results}

1) Behavioral Task Analysis: We performed a One way Anova analysis with the Training Condition as factor and the Reaction Time as Dependent Variable. The Result of Reaction Time is significant $(\mathrm{F}=4,009 ; \mathrm{p}=0,020)$; Post Hoc analysis shows a significant difference $(\mathrm{p}=0,016)$ between condition 2 and condition 3 (Leap Motion Training and Real Training in direction of slower Reaction Time in Leap Motion Condition (mean of $1.254 \mathrm{~ms}$ ) versus Real Condition (mean of $934 \mathrm{~ms}$ ). No effect on Reaction Time in Thinking Condition (mean $1.112 \mathrm{~ms})$.

2) ERP Analysis: We Performed a One way Anova analysis with the Training Condition as factor (1: Thinking training; 2: Leap Motion Training; 3 Real Training) and latencies and amplitude of N1, P1 and P3 ERP waves as dependent variables for the condition Affordance.

Main Results for N1 waves (Table 1): N1 shows significant values on $\mathrm{O} 1$ Latency $(\mathrm{F}=5,373 ; \mathrm{p}=0,012), \mathrm{O} 2$ Latency $(\mathrm{F}=5,570 ; \mathrm{p}=0,010)$ (Figure 6) and $\mathrm{Fz}$ Latency $(\mathrm{F}=5,206$; $\mathrm{p}=0,013$ ). Post Hoc Analysis, Bonferroni Test shows in O1 a significant difference between condition 1 (thinking training) and condition 2 (Leap Motion Training) with a significant value of $\mathrm{p}=0,025$ and a significant difference between condition 2 (Leap Motion Training) and Condition 3 (Real Training) with a significant value of $\mathrm{P}=0,030$. In $\mathrm{O} 2$ Latency, Bonferroni test shows a significant difference between 1 and $2(\mathrm{p}=0,044)$ and 2 and $3(\mathrm{p}=0,015)$ (Figure 8). In Fz Latency, Bonferroni test shows a significant difference between 1 and $2(\mathrm{p}=0,019)$ and 2 and $3(\mathrm{p}=0,053)$ (Figure 7). All the cortical activations are showed in Figure 8, 9, 10, 11, 12.

Main Results for P1 waves: P1 shows significant value on F4 Latency ( $\mathrm{F}=4,334$; $\mathrm{P}=0,025)$; Post Hoc Analysis, Bonfer-

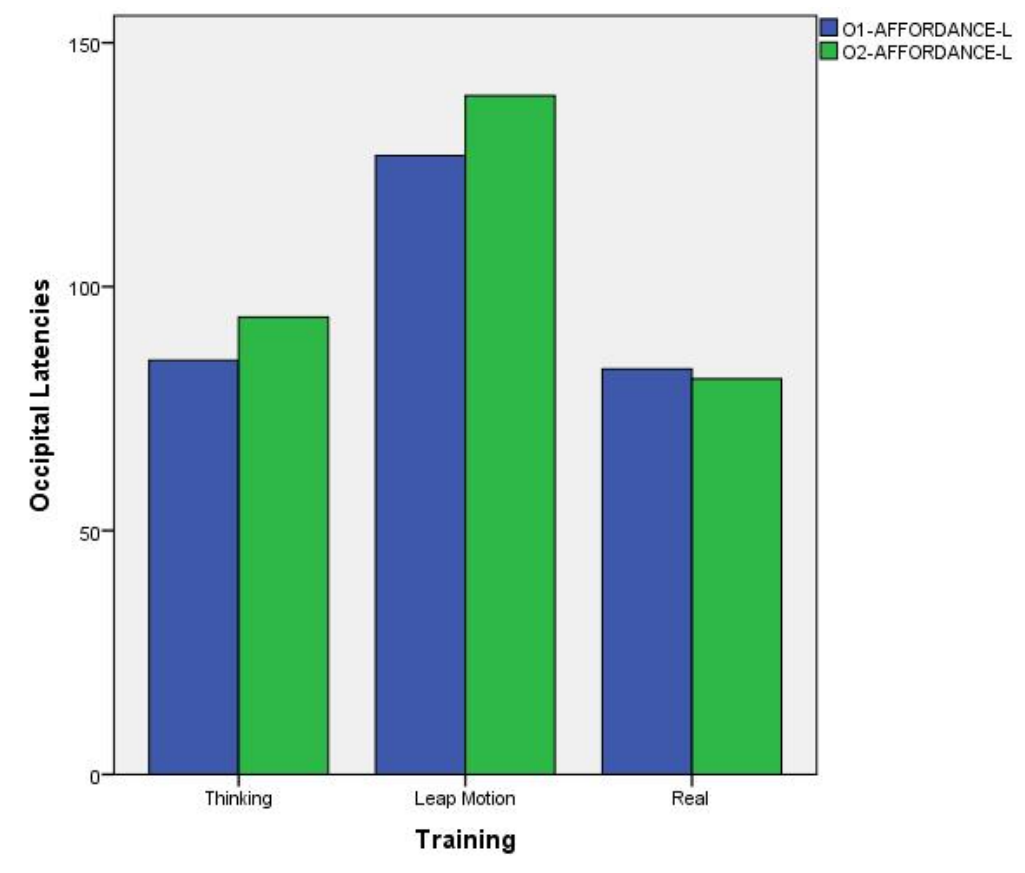

Figure 6. Occipital Latency

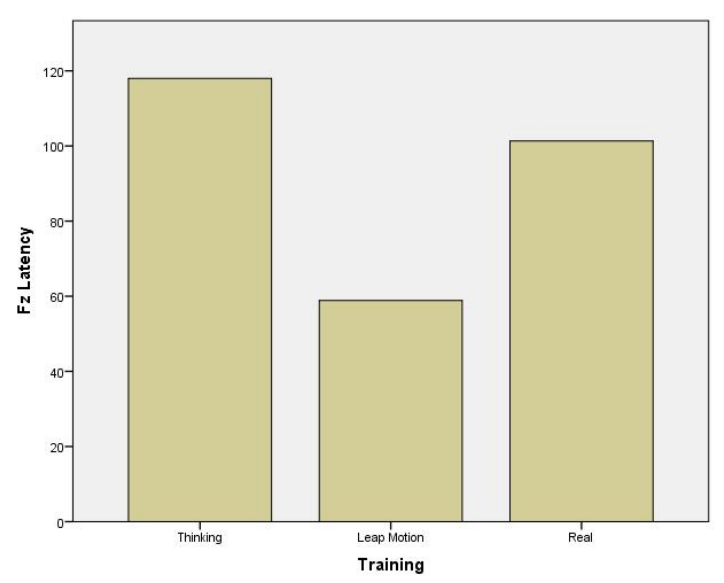

Figure 7. Frontal Latency

roni Test shows a significant difference between condition 1 and condition $3(\mathrm{P}=0,031)$. No significant difference in $\mathrm{P} 3$.

\section{E. Discussion of results}

Results of this work are very sensible to Virtual Leap Motion. In behavioral task we had a significant slower reaction

TABLE I

Anova Results and Post Hoc Analysis with Mean of Latencies

\begin{tabular}{|c|c|c|c|c|c|}
\hline N1 & TT1 L & TT2 L & TT3 L & F & p \\
\hline \hline$F z L$ & $113,30^{*}$ & $58^{*}$ & $107,75^{*}$ & 5,206 & 0,013 \\
\hline$O 1 L$ & $81,40^{*}$ & $128^{*}$ & $80,25^{*}$ & 5,373 & 0,012 \\
\hline$O 2 L$ & 89,40 & $137,56^{*}$ & $77,75^{*}$ & 5,570 & 0,010 \\
\hline P1 & TT1L & TT2L & TT3L & F & p \\
\hline$F 4 L$ & $100,20^{*}$ & 122,50 & $148,41^{*}$ & 4,334 & 0,025 \\
\hline
\end{tabular}




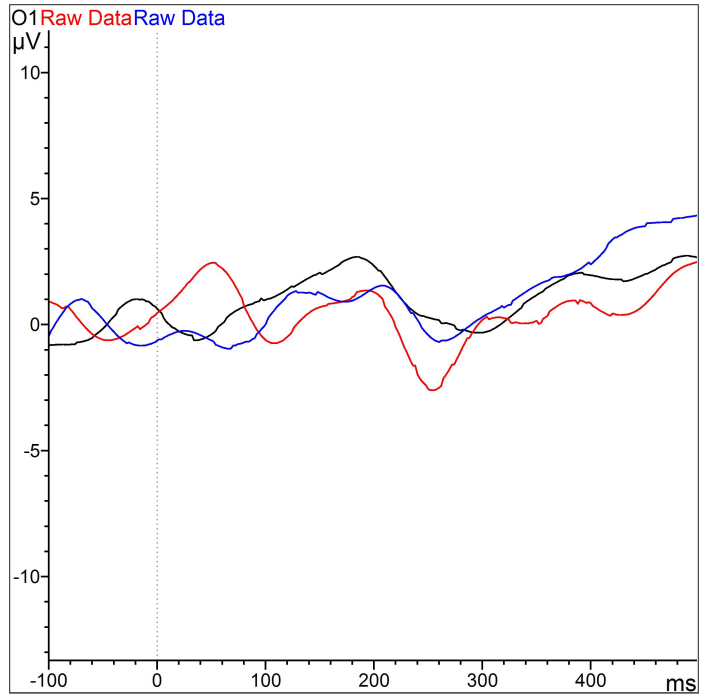

Figure 8. Matching ERP on O1 Channels: Black Line Thinking Training, Red Line Leap Motion Training, Blue Line Real Training on Affordance Images

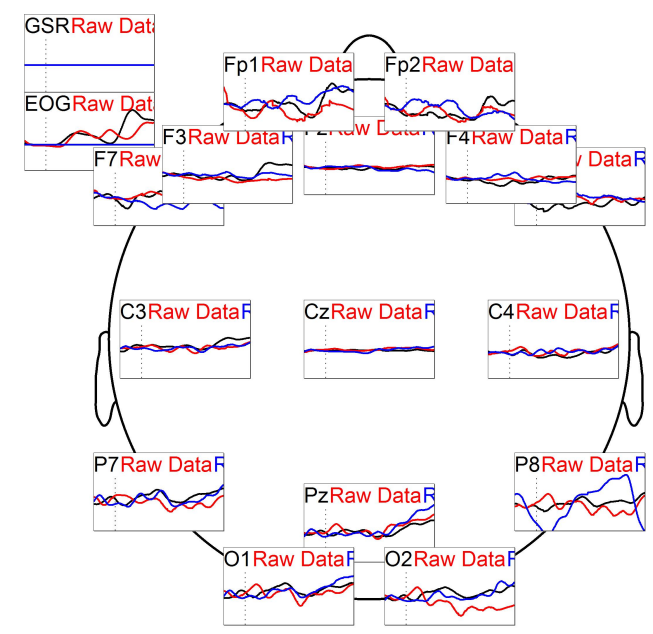

Figure 9. Head Matching ERP on 16 Positions Black Line Thinking Training, Red Line Leap Motion Training, Blue Line Real Training on Affordance Images

time in Leap Motion condition than in Real Condition. In Event Related Potentials result we found significant values in early components (N1 and P1). For N1 waves we had a slower latency in Occipital Channels and faster latency in Frontal Channel for Leap Motion Condition, while we found differences in P1 for Real Training Condition and Thinking Training Condition with a shorter latency in Frontal Right Channel only for Thinking Training Condition. No results in ERP Amplitude and no significant results in P3 component.

\section{CONCLUSIONS AND FUTURE WORKS}

Among the ERP's components we chose the most sensible to these conditions. N1 is a component of ERP that occurs between 50 and $150 \mathrm{~ms}$ and is considered too short to
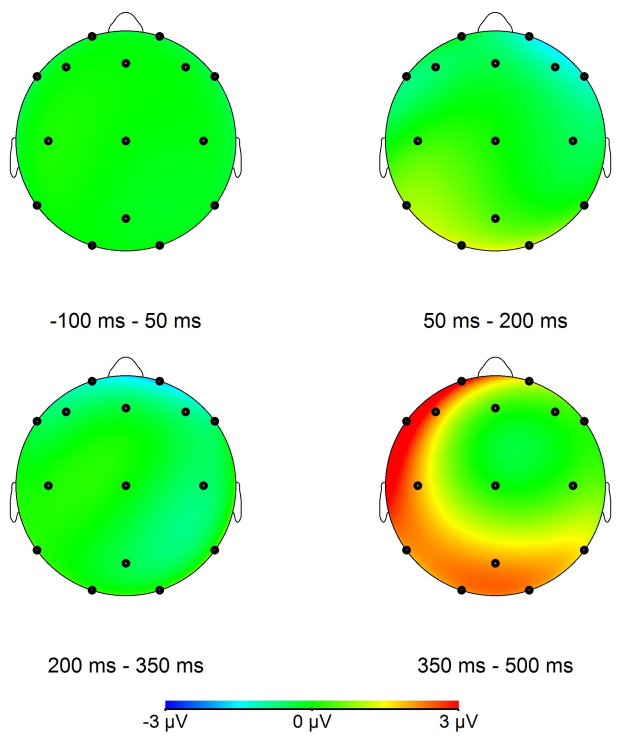

Figure 10. Arousal Post Thinking Training in Different ERP Components (Pre Stimulus, N1, P1 and P3)
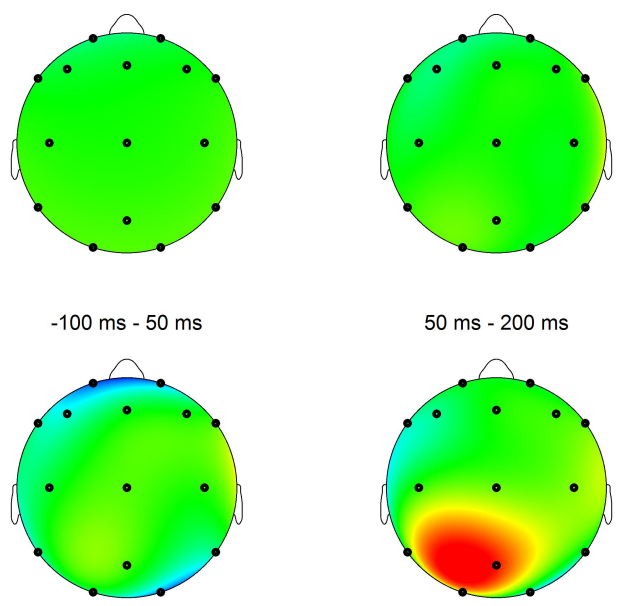

$$
\begin{array}{ccc}
200 \mathrm{~ms}-350 \mathrm{~ms} & & 350 \mathrm{~ms}-500 \mathrm{~ms} \\
-2.99 \mu \mathrm{V} & 0 \mu \mathrm{V} & 2.99 \mu \mathrm{V}
\end{array}
$$

Figure 11. Arousal Post Leap Motion Training in Different ERP Components (Pre Stimulus, N1, P1 and P3)

be influenced by top-down influences from the prefrontal cortex. Some researches show that sensory input is processed by the occipital cortex in $56 \mathrm{~ms}$ and that the information is communicated to the dorsolateral frontal cortex where it arrives in about $80 \mathrm{~ms}$ [17]. These higher-level areas create the attentive, repetition and arousal modulations upon the sensory area processing reflected in this component [18]. Another topdown influence upon $\mathrm{N} 1$ was suggested to be efference copies from a person's intended movements so that the stimulation deriving from them are not processed [19]. We can find another modulation when there is a created perturbation upon balance [20]. Instead, P1 is very sensible to attentional processing. 

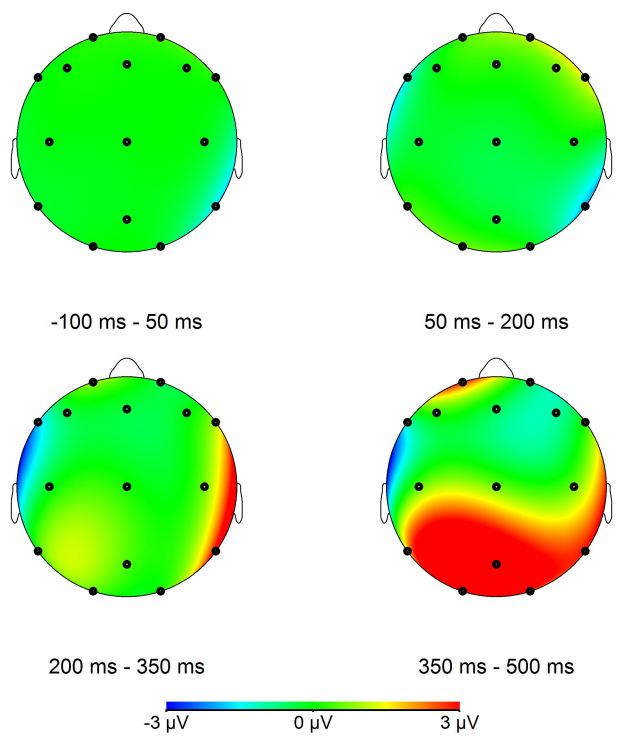

Figure 12. Arousal Post Real Training in Different ERP Components (Pre Stimulus, N1, P1 and P3)

In our results we found a sensible variation for latencies in these two components but not in the P3 component. This can happen because the sensory-motor and attentional processes are activated, in this experimental model, in a very early way, through the motor training and through the visual afforance of the objects. In behavioral results we have a slower reaction time in Leap Motion Condition. This could be due to the virtual motion training, that can be mostly considered a non integrated sensory multilevel training, because it involves both the thought feedback and the motor/visual feedback, without providing any tactile feedback: this might indeed somehow slow down the processes of motor response.

According to our result we can say that motion training, applied on interactive entertainment (in this experiment Leap Motion Training was used as an interactive entertainment), can significantly change the ability of discrimination and retention of the presented stimulus. In our study the imagined training and the real training can be sometimes in the same trend of results while the virtual training, in this case through the leap motion, visibly changes this trend. Early attentional components, in the occipital lobe, which is entrusted to visual sensory, increase latencies when the subject uses the leap motion. On the contrary, latencies decrease in frontal lobe, where the brain is involved in attentional arousal, sensorymotor action and action planning rather than visual perception. In this study we used grasping objects with motion of affordance, because they are more easy to try during a Real Training and because they had a interesting way of being perceived by cognition, because of objects have affordance related to action planning. Moreover, affordance allows to understand the motion way and the grasping way to manipulate the object. However, in some future experiments we will use the Leap Motion Controller with cognitive variables like Chess Learning, Anatomy Learning, Zoological Learning and
Emotional Learning (in a phobic situation) to study how a Virtual Motion Training with a Visual Feedback can allow Perception Processes, Emotional Processes and Learning. Further analysis could be done alongside the behavioral data on reaction times, the data on the level of entertainment and pleasure of the proposed conditions. All these results can be useful to implement in a neurocognitive and ergonomic way a technological and virtual product too.

\section{ACKNOWLEDGEMENTS}

This work has been supported by PON04a2_BEDOC@Work Project3.0 Education and Work in Cloud.

References for the Project: Prof. Antonio Domenico Grieco and Prof. Luca Mainetti, Department of Engineering for Innovation, University of Salento.

\section{REFERENCES}

[1] J. J. Gibson, "The theory of affordances," in Perceiving, Acting, and Knowing. Towards an Ecological Psychology. Hoboken, NJ: John Wiley \& Sons Inc., 1977, no. eds Shaw R., Bransford J.

[2] S. Thill, D. Caligiore, A. Borghi, T. Ziemke, and G. Baldassarre, "Theories and computational models of affordance and mirror systems: An integrative review," Neuroscience and Biobehavioral Reviews, vol. 37, no. 3, pp. 491-521, 2013.

[3] T. Handy, S. Grafton, N. Shroff, S. Ketay, and M. Gazzaniga, "Graspable objects grab attention when the potential for action is recognized," Nature Neuroscience, vol. 6, no. 4, pp. 421-427, 2003.

[4] E. Oztop, M. Kawato, and M. Arbib, "Mirror neurons and imitation: A computationally guided review," Neural Networks, vol. 19, no. 3, pp. 254-271, 2006.

[5] D. Gross, K. Stanney, and L. Cohn, "Evoking affordances in virtual environments via sensory-stimuli substitution," Presence: Teleoperators and Virtual Environments, vol. 14, no. 4, pp. 482-491, 2005.

[6] L. Bringoux, C. Bourdin, J.-C. Lepecq, P. Sandor, J.-M. Pergandi, and D. Mestre, "Interaction between reference frames during subjective vertical estimates in a tilted immersive virtual environment," Perception, vol. 38, no. 7, pp. 1053-1071, 2009.

[7] W. Warren Jr. and S. Whang, "Visual guidance of walking through apertures: Body-scaled information for affordances," Journal of Experimental Psychology: Human Perception and Performance, vol. 13, no. 3, pp. 371-383, 1987.

[8] S. J. Luck, An Introduction to the Event-related Potential Technique, 2nd ed. Cambridge, Mass.: MIT Press, 2014.

[9] L. Baolin, W. Zhongning, S. Guanjun, and W. J. Guangning, "Cognitive processing of traffic signs in immersive virtual reality environment: An ERP study," Neuroscience Letters, vol. 485, no. 1, pp. 43-48, 2010.

[10] U. Neumann and A. Majoros, "Cognitive, performance, and systems issues for augmented reality applications in manufacturing and maintenance," in Virtual Reality Annual International Symposium, 1998. Proceedings., IEEE 1998, 1998, pp. 4-11. [Online]. Available: http://graphics.usc.edu/cgit/pdf/papers/STAR-lib-app.pdf

[11] "The Leap Motion Controller." [Online]. Available: https://www.leapmotion.com

[12] F. Weichert, D. Bachmann, B. Rudak, and D. Fisseler, "Analysis of the accuracy and robustness of the Leap Motion Controller," Sensors (Switzerland), vol. 13, no. 5, pp. 6380-6393, 2013.

[13] K. Khoshelham and S. Elberink, "Accuracy and resolution of kinect depth data for indoor mapping applications," Sensors, vol. 12, no. 2, pp. 1437-1454, 2012

[14] "Introducing the Skeletal Tracking Model." [Online]. Available: https://developer.leapmotion.com/documentation/cpp/devguide/ Intro_Skeleton_API.html

[15] "Leap Motion App Store." [Online]. Available: https://apps.leapmotion.com

[16] "Inside Leap Motion: Designing Playground." [Online]. Available: http://blog.leapmotion.com/inside-leap-motion-designing-playground

[17] J. J. Foxe and G. V. Simpson, "Flow of activation from V1 to frontal cortex in humans," Experimental Brain Research, vol. 142, no. 1, pp. 139-150, 2002. 
[18] J. Coull, "Neural correlates of attention and arousal: insights from electrophysiology, functional neuroimaging and psychopharmacology," Progress in Neurobiology, vol. 55, no. 4, pp. 343 - 361, 1998.

[19] N. Kudo, K. Nakagome, K. Kasai, T. Araki, M. Fukuda, N. Kato, and A. Iwanami, "Effects of corollary discharge on event-related potentials during selective attention task in healthy men and women," Neuroscience Research, vol. 48, no. 1, pp. 59 - 64, 2004.

[20] G. Mochizuki, K. M. Sibley, H. J. Cheung, and W. E. McIlroy, "Cortical activity prior to predictable postural instability: Is there a difference between self-initiated and externally-initiated perturbations?" Brain Research, vol. 1279, no. 0, pp. 29 - 36, 2009.

[21] L. Garber, "Gestural Technology: Moving Interfaces in a New Direction [Technology News]," Computer, vol. 46, no. 10, pp. 22-25, October 2013.

[22] J. Guna, G. Jakus, M. Pogačnik, S. Tomažič, and J. Sodnik, "An analysis of the precision and reliability of the leap motion sensor and its suitability for static and dynamic tracking," Sensors (Switzerland), vol. 14 , no. 2, pp. 3702-3720, 2014

[23] "Leap Motion SDK and Plugin Documentation." [Online]. Available: https://developer.leapmotion.com/documentation

[24] M. Roussou, "Learning by Doing and Learning Through Play: An Exploration of Interactivity in Virtual Environments for Children," Comput. Entertain., vol. 2, no. 1, pp. 10-10, Jan. 2004.

[25] S. Jang, J. B. Black, and J. R. W., "Embodied Cognition and Virtual Reality in Learning to Visualize Anatomy," in Proceedings of the 32nd Annual Conference of the Cognitive Science Society (CogSci 2010), pp. 2326-2331. [Online]. Available: http://mindmodeling.org/cogsci2010/papers/0538/paper0538.pdf

[26] L. Shapiro, The Handbook of Embodied Cognition. Routledge, 2014.

[27] "Dune Suite Area DIM Data Processing and Immersive Environment." [Online]. Available: http://www.cetma.it/LinkClick.aspx?fileticket=m71Y0e5gjME\%3D $\&$ tabid $=200 \&$ language $=i t-I T$ 